\section{Antibacterial effect of Titanium alloys with Copper Nanoparticles: In vitro study.}

\section{Nataly Silva-Arriagada ${ }^{1}$, Andrés Gómez ${ }^{1}$, Marlene Muñoz ${ }^{1}$.}

Abstract: Objective: To evaluate the in-vitro antimicrobial effect of Titanium alloys with Copper Nanoparticles (CuNP) against Streptococcus mutans and Phorphyromonas gingivalis. Materials and Methods: An in vitro study was carried out. The unit of analysis corresponded to 10 healing abutments. In 5 abutments Copper nanoparticles (CuNP) electrodeposition was applied. The remaining 5 abutments corresponded to control. The healing abutments were then immersed in culture medium for $\mathcal{S}$. mutans and $P$. gingivalis for 14 days. Results: The agar plates with CuNP-coated abutments showed a lower growth, statistically significant for both bacterial strains. Conclusion: There is a statistically significant lower growth of $\mathcal{S}$. mutans and $P$. gingivalis in healing abutments with CuNP.

Keywords: Metal Nanoparticles; Copper; dental implant; Anti-Bacterial Agents; Dental materials.

\section{INTRODUCTION}

Caries and periodontal disease are two of the most prevalent diseases worldwide (Marcenes et al., 2013). In Chile, these diseases have a prevalence of around $98 \%$ in the population between 35-44 years old (Urzua et al., 2012). Among the preventive treatments for these diseases, the application of Copper nanoparticles (CuNP) on dental materials has been recently described (Hajipour et al., 2012; Zhang et al., 2013).

$\mathrm{Cu}$ appears as a material with high antibacterial levels and low toxicity, with biocidal properties and a role in the regeneration of endothelial and bone tissues (Zhang et al., 2013; Kruk et al., 2015; Zhang et al., 2015). Some studies have revealed that adding $\mathrm{Cu}$ to a Titanium alloy results in strong antibacterial activity, without reducing mechanical properties nor corrosion resistance (Vargas-Reus, 2012).

The antibacterial, antiviral and antifungal effect might derive from the size and elevated weight/volume proportion of the particles. In theory, such features should allow them to interact closely with the bacterial surface and its intracellular components (Kruk et al., 2015; Zhang et al., 2015; VargasReus, 2012; Allaker, 2010; Ren et al., 2009). The particles over 10nm would accumulate in the cellular membrane altering permeability and transport. On the other hand, the particles under $10 \mathrm{~nm}$ would penetrate the membrane, accumulate intracellularly and have an effect on the nucleic acids (Allaker, 2010; Ren et al., 2009).

The combination of $\mathrm{Cu}$ with Titanium or other medical and dental alloys has showed a great antibacterial effect on agar plates colonies count against Staphylococcus aureus and Escherichia coli (Zhang et al., 2013; Kruk et al., 2015; Allaker, 2010). This is relevant since bacterial infections following

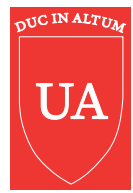

Affiliation: ${ }^{1}$ Facultad de Odontología, Universidad del Desarrollo, Concepción, Chile.

Corresponding: Nataly Silva-Arriagada, Barros Arana \#1735 Facultad de Odontología, Universidad del DesarrolIo, Concepción. Phone: +56984151517, E-mail: nsilvaa@gmail.com.

$\begin{array}{ll}\text { Receipt: } & 03 / 03 / 2019 \\ \text { Revised: } & 03 / 14 / 2019 \\ \text { Acceptance: } & 04 / 01 / 2019 \\ \text { Online: } & 04 / 28 / 2019\end{array}$

Conflict of interests: None.

Ethics approval: None.

Funding: None.

Authors' contributions: All authors carried out the entire study.

Acknowledgements: To Alphabio-tec ${ }^{\circledR}$ for donating the abutments.

doi: $10.32457 /$ ijmss.2018.035. 
implant placement are a significant source of complications (Canullo, 2015; Belibasakis, 2015). Therefore, bacterial infections associated with medical and dental metal alloys still pose a great challenge in the quality of healthcare.

In spite of this, there isn't enough information regarding the antibacterial potential of CuNP in dental implants abutments against oral bacteria. The aim of this study is to evaluate the in vitro antimicrobial effect of TiCuNP alloys against $S$. mutans and $P$. gingivalis.

\section{MATERIALS AND METHODS}

An in vitro pilot study was carried out. The unit of analysis was 10 Alphabio-tec ${ }^{\circledR}$ healing abutments made up of a Titanium, Aluminum, Vanadium alloy (Ti-6Al-4V). Electrodeposition of CuNP was applied to 5 abutments and the remaining 5 abutments were used as control.

Nanoparticle application protocol: At the Department of Materials Engineering of the Universidad de Concepción, 10 Alphabio-tec ${ }^{\circledR}$ healing abutments made up of a Titanium, Aluminum, Vanadium alloy (Ti-6Al-4V) were coated with CuNP. These CuNP were regular, with a size between 30 and $500 \mathrm{~nm}$, and were distributed homogenously across the healing abutment. The particles were obtained from metallic Copper, reproducing the chemical features of HG Copper (99,9\% pure grade $A$ cathode). An independent laboratory of the Faculty of Sciences of the Universidad de Concepción was in charge of corroborating the correct application of CuNP.

Electrodeposition of $\mathrm{Cu}$ on abutments was carried out using a 3-electrode setup, where a Platinum (Pt) plate was used as counter electrode and a standard Saturated Calomel Electrode (SCE) as reference electrode. Aluminum Vanadium (Ti-6Al-4V) dental abutments with an exposed area of $5.0 \mathrm{~mm} 2$ were used as working electrode. Prior to the experiment, these pieces were cleansed with alcohol and distilled water. The electrolyte bath contains $120 \mathrm{~mm}$ of CuS04-5H2O (Merck 99.9\%) and 1.8 M of H2SO4. The process was performed by galvanostatic electrodeposition applying a constant current of $-13,5 \mathrm{~mA}$ during 90 seconds. Immediately after electrodeposition, the samples were removed from the solution, washed with distilled water in order to remove non-adhered material and dried at room temperature.
Microbiological protocol: Freeze-dried strains reconstituted and cultivated at the Health Sciences laboratory of the Universidad del Desarrollo in Concepción were used.

S. mutans strain $\left(A T T C^{\circledR}\right.$ 25175TM) was spread over mitis salivarus agar $20 \%$ sacarose and then put into an anaerobiosis jar with a candle in its interior to create a microaerophilic environment for 48 hours at $37^{\circ} \mathrm{C}$ in order to promote bacterial growth.

P. gingivalis strain (ATTC ${ }^{\circledR}$ 33277TM) was spread over Columbia Blood agar and then put into an anaerobiosis jar, which contains gas generating envelopes for the production of an anaerobic atmosphere (GasPak) at $37^{\circ} \mathrm{C}$ during 14 days.

In order to cultivate each mentioned strain from broth to plates, $0.5 \%$ McFarland solution was prepared for McFarland visual scale. The purpose of this was to obtain a standard inoculum for each culture of $S$. mutans and $P$. gingivalis in physiological serum. This allowed the spreading of a standardized amount of bacteria ( $>108$ UFC) over each agar. For each strain, three replicas were made, obtaining a total of nine Petri dishes.

Two controls in two different plates were used in order to validate the used methodology. $0.12 \%$ Clorhexidine (CHX) was used as positive control and sterile distilled water as negative control.

The prepared plates were marked and placed under bell jar with sterile cotton swab in order to obtain a bacteria covered plate.

The Titanium healing abutments, with and without CuNP, were sterilized and inserted by pressing them into each one of the specific agar plates prepared from each studied bacterium and incubated during $48 \mathrm{hrs}$. in anaerobiosis jars at $37^{\circ} \mathrm{C}$ and $90 \%$ humidity.

After that, the antibacterial effect was observed through the presence of inhibition halo in the plates with S. mutans and $P$. gingivalis, according to inhibition zone width around the abutment measured in three points using a caliper. The width of the inhibition zone was calculated with the following formula.

Inhibition zone width = (inhibition width - abutment width) 2

Silva-Arriagada N, Gómez A, Muñoz M. Antibacterial effect of Titanium alloys with Copper Nanoparticles: In-vitro study. Int J Med Surg Sci. 2018; 5(4): 136-140. doi: 10.32457/ijmss.2018.035. 
In sterile test tube, $5 \mathrm{ml}$ mitis salivarus broth with $200 \mu \mathrm{l}$ bacterium (S.mutans) and $5 \mathrm{ml}$ Columbia broth with $200 \mu \mathrm{l}$ bacterium (P.gingivalis) were inoculated. These mixtures were incubated at $37^{\circ} \mathrm{C}$ for $48 \mathrm{hrs}$. in culture oven. For each test tube, three replicas of each bacterial strain were made.

$1 \mathrm{ml}$ of $100 \%$ mixture was extracted and put over a Neubauer chamber, the sample was observed with CX 21 Olympus ${ }^{\circledR}$ microscope at $100 x$ magnification. Three grids of the Neubauer chamber counting area were randomly selected and the bacteria present in each one of the $S$. mutans and $P$. gingivalis strains were directly counted.

A random count of the selected three areas was performed, obtaining an average, which was then multiplied by 103 in order to convert the values according to the chamber used.

Statistical analysis: The data obtained were analyzed using Stata 14.0 software (StataCorp), with 95\% confidence interval and 5\% significance level. For descriptive analysis, the position, central tendency and variability statistics were used, in which maximum and minimum values, median, mean and standard deviation were considered. For inferential analysis, the normality was evaluated through Shapiro Wilk test, showing nonparametric distribution; therefore, Wilcoxon hypothesis tests were used.

\section{RESULTS}

Table 1 shows bacterial growth of $S$. mutans and $P$. gingivalis in abutments with and without CuNP $(p<0.05)$.

\section{DISCUSSION}

This study revealed an inhibition of bacterial growth of $S$. mutans and $P$. gingivalis in abutments with CuNP. These findings agree with those reported by Amiri et al. (2017), where an inhibition of bacterial growth of $S$. mutans in solutions of Copper oxide NP was observed, and by VargasReus et al. (2012), which also showed inhibition of growth in bacteria associated with peri-implantitis, such as $P$. gingivalis.

Currently, there is a tendency to strengthen biomaterials by incorporating components that reduce the risks of infection and foster tissue regeneration. Among these components, $\mathrm{Cu}$, and particularly CuNP, have gained recognition as a complementary material to polymers and alloys already used in the health area, such as polypropylene, polystyrene and $\mathrm{Ti}$ alloys, among others. Actually, it is estimated that these polymers and alloys could benefit from the features of $\mathrm{Cu}$, which include: bone regeneration, angiogenic ability, anti-inflammatory characteristics and antimicrobial features, among others (Zhang, 2013; Allaker \& Memarzadeh, 2014; Finney et al., 2009; Gérard et al., 2010; Barralet et al., 2009). The advantage of using CuNP, instead of proper $\mathrm{Cu}$, is that it allows using less mineral amount, reducing the cost of its potential applications. Additionally, it would allow reducing the presence of potential resistance, corrosion, galvanism and cytotoxicity on cells. To sum up, reducing the concentration of the Copper mineral should have a positive impact on its biocompatibility.

Even though the biocompatibility of Copper has

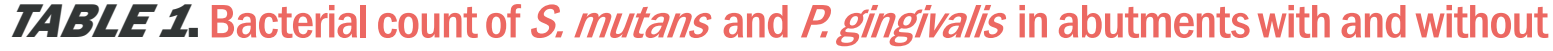 nanoparticles.}

\begin{tabular}{ccccccc} 
Bacteria & Abutment & Minimum & Maximum & Median & Mean \pm SD* & $p$ - value \\
& Without CuNP & $3 \times 10^{7}$ & $3.32 \times 10^{7}$ & $3.12 \times 10^{7}$ & $3.15 \times 10^{7} \pm 1.62 \times 10^{6}$ & \\
S. mutans & With CuNP & 0 & $2.4 \times 10^{6}$ & $1.6 \times 10^{6}$ & $1.33 \times 10^{6} \pm 1.22 \times 106$ & 0.0495 \\
& Without CuNP & $2.56 \times 10^{7}$ & $2.92 \times 10^{7}$ & $2.6 \times 10^{7}$ & $2.69 \times 10^{7} \pm 1.97 \times 10^{6}$ & \\
\multirow{2}{*}{ P. gingivalis } & With CuNP & 0 & $1.2 \times 10^{6}$ & $0.4 \times 10^{6}$ & $0.53 \times 10^{6} \pm 0.61 \times 10^{6}$ & 0.0495
\end{tabular}

*Standard Deviation

Silva-Arriagada N, Gómez A, Muñoz M. Antibacterial effect of Titanium alloys with Copper Nanoparticles: In-vitro study. Int J Med Surg Sci. 2018; 5(4): 136-140. doi: 10.32457/ijmss.2018.035. 
been proven, with less cytotoxicity than Silver and higher resistance to corrosion, adding Silver Nanoparticles on $\mathrm{Ti}$ surfaces as antimicrobial agent has been widely reported and few studies have considered adding CuNP for odontological use (Zhang et al., 2015; Shirai et al., 2009; Ferraris \& Spriano, 2016).

Our results show an antibacterial effect against oral pathogens, confirming what has been previously reported on fixed orthodontic appliances and Clorhexidine-based mouthwash (Ramazanzadeh et al., 2015; Ahrari et al., 2015). However, the antibacterial effect of CuNP on TI alloy used for dental implants has been scarcely studied. The only findings in this field are against oral bacteria that are not classically linked to caries and periodontal/peri-implant disease, such as E. coli and S. aureus (Zhang et al., 2013).

This study is, therefore, one of the first that analyzes its effect against $S$. mutans and $P$. gingivalis.

The addition of CuNP appears as an innovative alternative, although up to date, all reported findings regarding dental use of CuNP have been developed on in vitro studies. Furthermore, regarding this and other surface treatments to improve antimicrobial properties on dental implants, it is worth noticing that one of the issues concerning this strategy is that it offers a high initial release of the agent, decreasing rapidly in the following days. Also, while with higher concentrations there is a greater effect, it must be noticed that with high concentrations there might be cytotoxical behavior (Ferraris \& Spriano, 2016). Nevertheless, gynecology has been using copper-based intrauterine contraceptive devices since the middle of the last century, with a high margin of safety.

Some limitations of this study are the reduced sample size and its in vitro nature. It is, thus, necessary to carry out further studies with a bigger sample size, using a substrate that is more similar to oral biofilm and recreating the conditions of the healing abutments in the oral cavity. After that, in vivo studies could be carried out.

\section{CONCLUSION}

The results showed that bacterial growth of $S$. mutans and $P$. gingivalis was statistically lower on Titanium abutments with added CuNP.

\section{REFERENCES}

Ahrari F, Eslami N, Rajabi 0, Ghazvini K, Barati S. The antimicrobial sensitivity of Streptococcus mutans and Streptococcus sangius to colloidal solutions of different nanoparticles applied as mouthwashes. Dent Res J (Isfahan). 2015; 12(1): 44-9.

Allaker RP. The use of nanoparticles to control oral biofilm formation. J Dent Res. 2010; 89(11): 1175-1186.

Allaker RP, Memarzadeh K. Nanoparticles and the control of oral infections. Int J Antimicrob Agents. 2014;43(2):95-104.

Amiri M, Etemadifar Z, Daneshkazemi A, Nateghi M. Antimicrobial effect of copper oxide nanoparticles on some oral bacteria and candida species. J Dent Biomater. 2017;4(1): 347-352.

Barralet J, Gbureck U, Habibovic P, Vorndran E, Gerard C, Doillon CJ. Angiogenesis in calcium phosphate scaffolds by inorganic copper ion release. Tissue Eng Part $A$. 2009;15(7):1601-9.

Belibasakis GN, Charalampakis G, Bostanci N, Stadlinger B. Peri-implant infections of oral biofilm etiology. In Biofilmbased Healthcare-associated Infections. Switzerland: Springer International Publishing; 2015. 69-84.

Canullo L, Rossetti PH, Penarrocha D. Identification of Enterococcus Faecalis and Pseudomonas Aeruginosa on and in Implants in Individuals with Peri-implant Disease: A Cross-Sectional Study. Int J Oral Maxillofac Implants. 2015; 30(3):583-7.

Ferraris S, Spriano S. Antibacterial titanium surfaces for medical implants. Mater Sci Eng C Mater Biol Appl. 2016;61:965-978.

Finney L, Vogt S, Fukai T, Glesne D. Copper and angiogenesis: unravelling a relationship key to cancer progression. Clin Exp Pharmacol Physiol. 2009; 36(1): 88-94.

Gérard C, Bordeleau LJ, Barralet J, Doillon CJ. The stimulation of angiogenesis and collagen deposition by copper. Biomaterials. 2010; 31(5):824-831.

Hajipour MJ, Fromm KM, Ashkarran AA, de Aberasturi DJ, de Larramendi IR, Rojo T, Serpooshan V, Parak WJ, Mahmoudi M. Antibacterial properties of nanoparticles. Trends biotechnol. 2012; 30(10): 499-511.

Kruk T, Szczepanowicz K, Stefańska J, Socha RP, Warszyński P. Synthesis and antimicrobial activity of monodisperse copper nanoparticles. Colloids Surf B Biointerfaces. 2015; 128: 17-22.

Marcenes W, Kassebaum NJ, Bernabé E, Flaxman A, Naghavi $M$, Lopez A, Murray CJ. Global burden of oral conditions in 19902010: a systematic analysis. J Dent Res. 2013;92(7):592-7.

Ramazanzadeh B, Jahanbin A, Yaghoubi M, Shahtahmassbi N, Ghazvini K, Shakeri M, Shafaee H. Comparison of antibacterial effects of $\mathrm{ZnO}$ and $\mathrm{CuO}$ nanoparticles coated brackets against Streptococcus mutans. J Dent (Shiraz). 2015; $16(3): 200-5$.

Ren G, Hu D, Cheng EW, Vargas-Reus MA, Reip P, Allaker RP. Characterisation of copper oxide nanoparticles for 
antimicrobial applications. Int J Antimicrob Agents. 2009; 33(6): 587-590.

Shirai T, Tsuchiya H, Shimizu T, Ohtani K, Zen Y, Tomita K. Prevention of pin tract infection with titanium-copper alloys. J Biomed Mater Res B Appl Biomater. 2009; 91(1):373-380.

Urzua I, Mendoza C, Arteaga 0, Rodríguez G, Cabello R, Faleiros S, Carvajal P, Muñoz A, Espinoza I, Aranda W, Gamonal J. Dental caries prevalence and tooth loss in chilean adult population: first national dental examination survey. Int J Dent. 2012;2012: 810170

Vargas-Reus MA, Memarzadeh K, Huang J, Ren GG, Allaker RP. Antimicrobial activity of nanoparticulate metal oxides against peri-implantitis pathogens. Int J Antimicrob Agents. 2012;40(2):135-139.

Zhang E, Li F, Wang H, Liu J, Wang C, Li M, Yang K. A new antibacterial titanium-copper sintered alloy: preparation and antibacterial property. Mater Sci Eng C Mater Biol Appl. 2013; 33(7): 4280-4287.

Zhang E, Zheng L, Liu J, Bai B, Liu C. Influence of Cu content on the cell biocompatibility of $\mathrm{Ti}-\mathrm{Cu}$ sintered alloys. Mater Sci Eng C Mater Biol Appl. 2015;46:148-57. 